\title{
Association between cranial asymmetry severity and chronic subdural hematoma laterality
}

Cheng-Ta Hsieh, MD, Chih-Ta Huang, MD, Yu-Hao Chen, MD, Jui-Ming Sun, MD.

\begin{abstract}

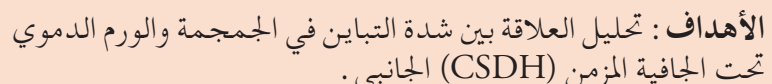

الطريقة: أجرينا دراسة بأثر رجعي لتقييم 120 مريضا يعانون التبانيا

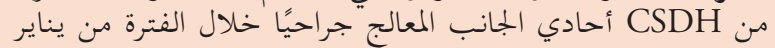

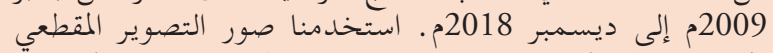

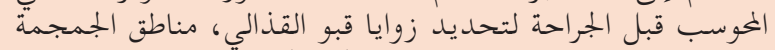

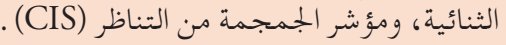

النتائج : كان الجنس الذكري (70\%) هو العامل السائد في تعزيز

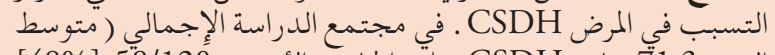

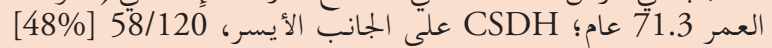

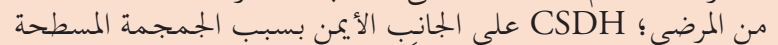

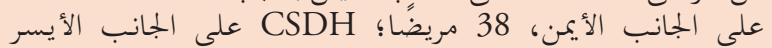

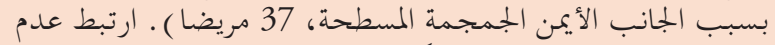

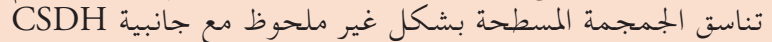

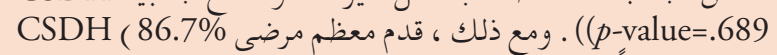

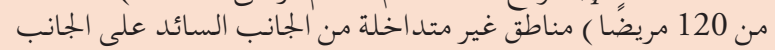

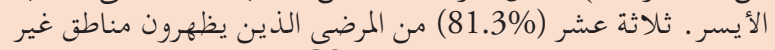

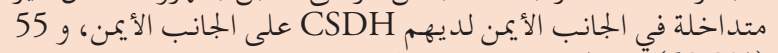

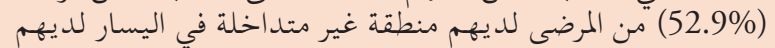
CSDH

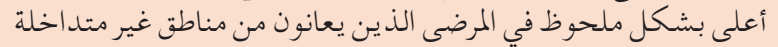

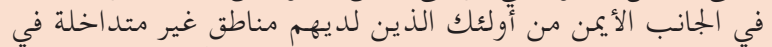

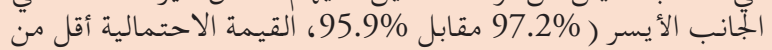
. (0.0001

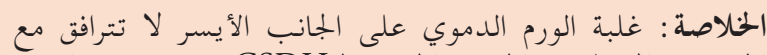

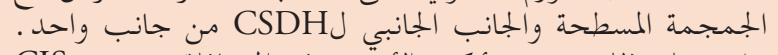

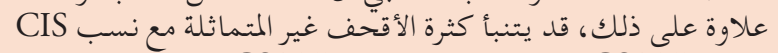

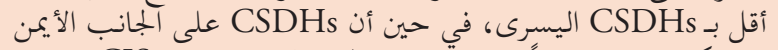

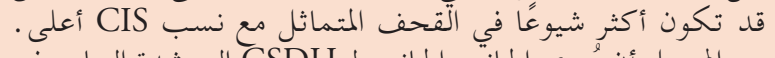

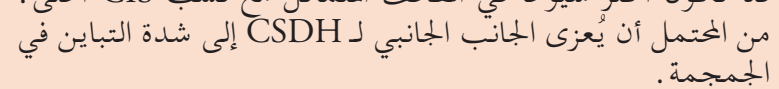

Objectives: To analyze the association between cranial asymmetry severity and chronic subdural hematoma $(\mathrm{CSDH})$ laterality.

Methods: We retrospectively assessed 120 patients with surgically treated unilateral CSDH from January
2009 to December 2018. Preoperative computed tomography images were used to determine occipital vault angles, bilateral cranium areas, and cranial index of symmetry (CIS) ratios.

Results: The male sex (70\%) was the predominant factor promoting $\mathrm{CSDH}$ pathogenesis. In the overall study population (mean age, 71.3 years; left-sided $\mathrm{CSDH}, 58 / 120$ [48\%] patients; right-sided CSDH due to right-sided flat cranium, 38 patients; leftsided $\mathrm{CSDH}$ due to right-sided flat cranium, 37 patients). Flat cranial asymmetry was nonsignificantly associated with $\mathrm{CSDH}$ laterality $(p$-value $=.689)$. However, most CSDH patients $(86.7 \%$ of 120 patients) presented dominant-sided nonoverlapping areas on the left side. Thirteen $(81.3 \%)$ patients presenting right-dominant nonoverlapping areas had right-sided CSDH, and 55 (52.9\%) patients had leftdominant nonoverlapping area had left-sided CSDH $(p$-value $=0.01)$. The CIS ratio was significantly higher in patients with right-dominant nonoverlapping areas than in those with left-dominant nonoverlapping areas $(97.2 \%$ vs $95.9 \%, \quad p$-value $<0.0001)$.

Conclusion: Left-sided hematoma predominance is not associated with a flat cranium and laterality of unilateral CSDH. Moreover, more asymmetric crania with lower CIS ratios may predict left-sided $\mathrm{CSDH}$, whereas the right-sided $\mathrm{CSDH}$ s may be more common in symmetric crania with higher CIS ratios. The CSDH laterality is potentially attributable to cranial asymmetry severity.

Neurosciences 2020; Vol. 25 (3): 205-209 doi: 10.17712/nsj.2020.3.20190125

From the Division of Neurosurgery (Hsieh, Huang), Department of Surgery, Sijhih Cathay General Hospital, and the Department of Medicine (Hsieh, Huang), School of Medicine, Fu Jen Catholic University, from the Department of Neurological Surgery (Hsieh, Sun), Tri-Service General Hospital, National Defense Medical Center, Taipei, from the Section of Neurosurgery (Chen, Sun), Department of Surgery, Chia-Yi Christian Hospital, from Chung-Jen Junior College of Nursing (Chen), Health Sciences and Management, Chia-Yi, and from the Department of Biotechnology (Sun), Asia University, Taichung, Taiwan.

Received 22nd December 2019. Accepted 24th February 2020.

Address correspondence and reprint request to: Dr. Jui-Ming Sun, Section of Neurosurgery, Department of Surgery, Chia-Yi Christian Hospital, Chia-Yi, Taiwan. E-mail: nogor@mail2000.com.tw ORCID ID: https://orcid.org/0000-0002-2018-7015 
$\mathrm{C}$ hronic subdural hematoma $(\mathrm{CSDH})$, a common neurosurgical entity, is considered a delayed manifestation of head trauma, occurring mostly in older men. ${ }^{1}$ Although CSDHs can occur in unilateral or bilateral intracranial spaces, left-sided predominance has been previously reported. ${ }^{2,3}$ Few studies have reported that cranial morphology and gravity contribute to the laterality of $\mathrm{CSDH}^{4-6}$ Cranial asymmetry is more common among crania flattened toward the right side, influencing the left-sided predominance of CSDH. ${ }^{6}$ The cranial index of symmetry (CIS) with a semiautomated method has been introduced to objectively determine the severity of cranial deformations including plagiocephaly. ${ }^{7}$ A preliminary study reported that the objective nonradiographic method potentially serves as an unbiased measurement of cranial asymmetry. ${ }^{7}$ The flat side has been simply defined by a smaller angle through a simple method considering angles among 3 lines. ${ }^{4-6}$ However, the angles may be manually affected by the points of the intersection of the manually selected lines, thus obstructing cranial asymmetry assessment. Therefore, here, we aimed to clarify the association between the severity of cranial asymmetry and the laterality of $\mathrm{CSDH}$ by using an objective semiautomated method.

Methods. Patient selection. In this retrospective study, we reviewed the medical records of patients who underwent surgical treatment for CSDH at our institute from January 2009 to December 2018. Our study was approved by the Institutional Review Board of Cathay General Hospital in Taiwan (CGH-P108069). The inclusion criteria were as follows: (1) $\mathrm{CSDH}$ confirmed through brain computed tomography (CT) without contrast enhancement, (2) presentation of only unilateral CSDH with neurological symptoms, and (3) age $>20$ years. The exclusion criteria were as follows: (1) concomitant occurrence of other types of traumatic brain injury; (2) performance of previous neurosurgical procedures including craniectomy, craniotomy, or shunting procedures; (3) development from nontraumatic etiologies such as vascular abnormalities or

Disclosure. Authors have no conflict of interests, and the work was not supported or funded by any drug company. This study was approved by the Institutional Review Board of Cathay General Hospital in Taiwan (CGH-P108069). neoplasm; and (4) missing data or images. In total, 120 patients with unilateral CSDH were enrolled.

Radiological evaluation. Preoperative CT images of all patients were obtained to assess cranial symmetry and the location of CSDH. Cranial asymmetry in axial view of CT images was defined using 2 methods: the conventional angle method and the objective semiautomated method developed here.

In the angle method (Figure 1), cranial asymmetry was investigated using 3 lines passing the midline bilaterally along the cranium, as previously described. ${ }^{4,6}$ The flat side was the side of the smaller angle. Cranial "asymmetry" was defined as a difference of $>2^{\circ}$ in both angles. In the objected semiautomated method developed here, cranial asymmetry was analyzed in accordance with the concept described by Zonenshayn et al. ${ }^{7}$ The image analysis software was developed on the basis of the LabVIEW system (National Instruments, Texas, USA). CT images were loaded into this software system. The nasion and inion, manually denoted by black spots, were considered the midline. The subsequent analysis was automated, including subtraction of the skull bone, encoding of the number of pixels, and determination of each cranial area. Considering the midline as the axis, one side of the cranium could be horizontally flipped onto the other side to determine the overlapping and nonoverlapping areas (Figure 2). The dominant nonoverlapping side was defined as the side of the larger nonoverlapping area. The CIS ratio was determined as twice the overlapping area divided by the total area.

Statistical analysis. Statistical analyses were performed using SPSS (version 22.0). The data are presented as means and their standard deviations. Between-group comparisons were performed using the independent-samples Student t test, Fisher exact test, and cross-table analysis. Differences were considered statistically significant when $p$-value $<0.05$.

Results. Of the 120 patients enrolled, male sex (70\%) had unilateral CSDH. The mean age of the entire study population was $71.3 \pm 12.9$ (range, 36-98) years. However, only 58 (48\%) patients had left-sided $\mathrm{CSDH}$.

Based on CSDH laterality, all patients were divided into 2 groups: right-sided and left-sided $\mathrm{CSDH}$ (Table 1). Of the 79 patients cranial asymmetry defined as a difference of $>2^{\circ}$ in both angles, we analyzed the association between the laterality of $\mathrm{CSDH}$ s and the side of flat cranium. Among 53 patients with right-sided flat cranium, there were 27 right-sided CSDH and 26 left-sided CSDH. Among 26 patients with left-sided flat cranium, 14 hematomas occurred in right side and 
12 hematomas occurred in left side. This association was not significant $(p=0.689)$.

By using the objective semiautomated method, the areas of total hemisphere, right hemisphere, and left hemisphere in the right-sided CSDH group were similar to those in theleft-sided CSDH group (Table2). However, patients with right-sided CSDH had a significantly larger right-sided nonoverlapping area than did those with left-sided CSDH $(p=0.002)$. Furthermore, the left-sided nonoverlapping area was significantly larger in patients with left-sided $\mathrm{CSDH}$ than in those with right-sided CSDH ( $p=0.003)$. The CIS ratio was slightly but nonsignificantly higher in patients with right-sided $\mathrm{CSDH}$ than in those with left-sided CSDH $(p=0.05)$.

Based on the dominant side of the nonoverlapping area, patients were divided into right-and left-dominant nonoverlapping groups (Table 3). Among $120 \mathrm{CSDH}$ patients, most patients (86.7\%) displayed left-sided predominance in the nonoverlapping area. The mean age

Table 1 - Summary of chronic subdural hematoma laterality and cranial asymmetry in the patients.

\begin{tabular}{lccc}
\hline Variables & \multicolumn{3}{c}{ Hematoma side } \\
& Right $(\mathbf{n}=62)$ & Left $(\mathbf{n}=58)$ & $P$-value \\
\hline Age & $69.3 \pm 12.9$ & $73.4 \pm 12.7$ & 0.085 \\
Gender & & & 0.524 \\
Female & 17 & 19 & \\
Male & 45 & 39 & \\
Cranial morphology & & & 0.944 \\
Symmetry & 21 & 20 & \\
Asymmetry & 41 & 38 & \\
Flat side & & & 0.777 \\
Right & 38 & 37 & \\
Left & 24 & 21 & \\
\hline
\end{tabular}

Table 2 - Summary of cranial areas and chronic subdural hematoma laterality.

\begin{tabular}{lccc}
\hline Variables & \multicolumn{2}{c}{ Hematoma side } \\
& Right $(\mathrm{n}=62)$ & Left $(\mathrm{n}=58)$ & $P$-value \\
\hline Cranial area $\left(\mathrm{mm}^{2}\right)$ & & \\
Total & $16212 \pm 2549$ & $16080 \pm 2109$ & 0.759 \\
Right side & $7984 \pm 1290$ & $7767 \pm 994$ & 0.306 \\
Left side & $8228 \pm 1290$ & $8313 \pm 1171$ & 0.706 \\
overlapping & $17894 \pm 3083$ & $17830 \pm 2886$ & 0.907 \\
Non-overlapping & & & \\
right & $389 \pm 402$ & $187 \pm 286$ & 0.002 \\
left & $934 \pm 746$ & $1437 \pm 1038$ & 0.003 \\
CIS ratio (\%) & $96.5 \pm 1.8$ & $95.7 \pm 2.5$ & 0.05 \\
\hline \multicolumn{4}{c}{ CIS - cranial index of symmetry } \\
\hline
\end{tabular}

and sex gender between 2 groups were not significantly different. Moreover, the association among the dominant nonoverlapping area, flat cranium, and asymmetric cranial morphology was not significant. The areas of the total, right side, left side, and overlapping regions were similar in both groups. 13 (81.3\%) of 16 patients with right-dominant nonoverlapping areas had right-sided CSDH, where 55 (52.9\%) of 104 patients had left-sided $\mathrm{CSDH}$. The dominant-sided nonoverlapping area was significantly associated the same-sided hematoma $(p=0.011)$. Furthermore, the CIS ratio was significantly higher in patients with right-dominant nonoverlapping areas than in those with left-dominant nonoverlapping areas $(p<0.0001)$. As the abovementioned results, high cranial asymmetry with a low CIS ratio may predict left-sided CSDH (Figure 3A and B), whereas right-sided $\mathrm{CSDH}$ is potentially more common among symmetric crania with a high CIS ratio (Figure 3C).

Discussion. The $\mathrm{CSDH}$ is a well-documented neurosurgical entity, mostly occurring among older people. The incidence of CSDH has increased worldwide because of progressive aging of the population, a lower

Table 3 - Summary of patients with chronic subdural hematoma based on dominant nonoverlapping areas.

\begin{tabular}{|c|c|c|c|}
\hline \multirow[t]{2}{*}{ Variables } & \multicolumn{3}{|c|}{ Non-overlapping dominant side } \\
\hline & Right $(n=16)$ & Left $(n=104)$ & $P$-value \\
\hline Age & $69.1 \pm 14.8$ & $71.6 \pm 12.6$ & 0.462 \\
\hline Gender & & & 0.907 \\
\hline Female & 5 & 31 & \\
\hline Male & 11 & 73 & \\
\hline Hematoma side & & & 0.011 \\
\hline Right & 13 & 49 & \\
\hline Left & 3 & 55 & \\
\hline Flat side & & & 0.267 \\
\hline Right & 8 & 67 & \\
\hline Left & 8 & 37 & \\
\hline Cranial morphology & & & 0.385 \\
\hline Symmetry & 7 & 34 & \\
\hline Asymmetry & 9 & 70 & \\
\hline \multicolumn{4}{|l|}{ Brain area $(\mathrm{mm} 2)$} \\
\hline Total & $15952 \pm 2757$ & $16178 \pm 2281$ & 0.72 \\
\hline Right & $8118 \pm 1423$ & $7842 \pm 1114$ & 0.377 \\
\hline Left & $7834 \pm 1341$ & $8336 \pm 1205$ & 0.129 \\
\hline overlapping & $17967 \pm 3401$ & $17845 \pm 2925$ & 0.882 \\
\hline \multicolumn{4}{|l|}{ Non-overlapping } \\
\hline right & $822 \pm 361$ & $210 \pm 288$ & $<0.001$ \\
\hline left & $180 \pm 148$ & $1331 \pm 905$ & $<0.001$ \\
\hline CIS Ratio (\%) & $\frac{97.2 \pm 0.9}{\text { inial index of } s}$ & $\frac{95.9 \pm 2.3}{\text { nmetry }}$ & $<0.001$ \\
\hline
\end{tabular}




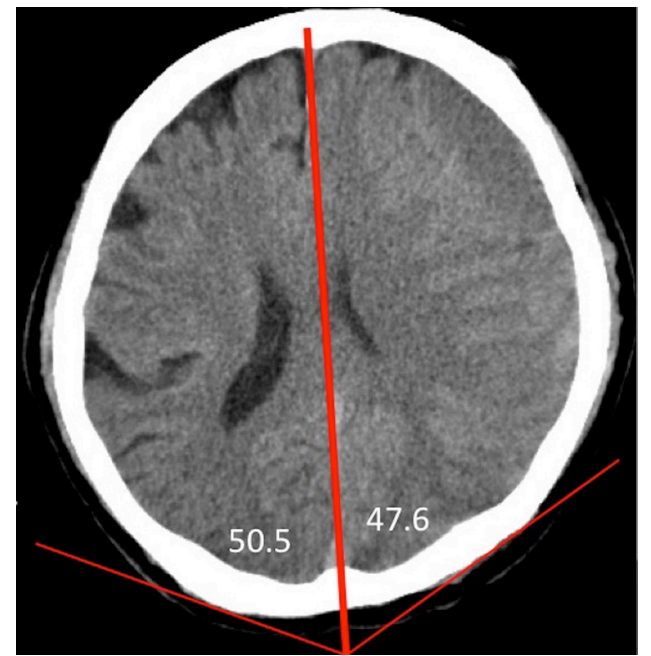

Figure 1 - Angle method, involving 3 lines traversing the midline and both sides of the cranium.

imaging threshold for patients with head trauma, and increased use of anti-coagulants or antiplatelet agents. ${ }^{8-10}$ Brain atrophy, hemorrhage from a tear in the bridging vein after initial heat trauma, and local inflammation or angiogenesis owing to hematoma markedly contribute to CSDH pathogenesis in older people. ${ }^{110-12}$ The CSDH with left-sided predominance has been described previously. ${ }^{2,3}$ Nayil et al ${ }^{3}$ retrospectively reviewed 863 patients with unilateral $\mathrm{CSDH}$ and reported that the hematoma was left-sided in $55.9 \%$ of cases. Of 215 patients with CSDHs, MacFarlane et al ${ }^{2}$ reported 123 (57\%) patients with left-sided CSDH. Here, most patients were male $(70 \%)$ and older with a mean age of 71.3 years. However, only 58 (48\%) of 120 patients had left-sided CSDH, in contrast to previous reports. ${ }^{2,3}$

The reason underlying this left-sided predominance in $\mathrm{CSDH}$ is unclear. Cranial morphology may be associated with $\mathrm{CSDH}$ laterality. ${ }^{2,4-6}$ On using the conventional angle method, bilateral CSDHs were frequently observed in patients with cranial asymmetry, whereas $\mathrm{CSDH}$ was more commonly located at the region opposite the skull toward the flat side. ${ }^{4} \mathrm{Kim}$ et $\mathrm{al}^{6}$ retrospectively assessed 182 patients with $\mathrm{CSDH}$ and reported that in the right flat cranium, hematoma more commonly occurred on the opposite side of the flat side. Among patients with left flat crania, $\mathrm{CSDH}$ was more common on the same side. However, Akhaddar et $\mathrm{al}^{5}$ assessed the angles in the frontal and occipital vault and reported that in patients with asymmetric crania, hematoma was more commonly present on the same side of the most curved frontal or occipital convexity. These results are in contrast to those of Kim et al. ${ }^{6}$ Here,
Right cranium

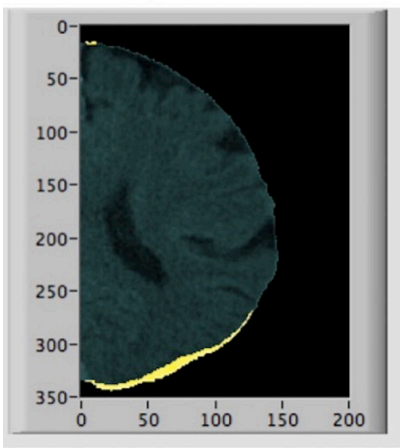

Left cranium

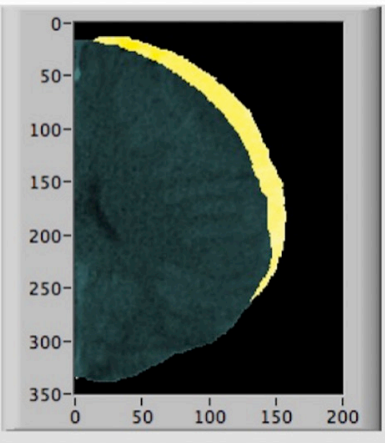

Figure 2 - Objective semiautomated method (developed here) to assess the cranial cross-sectional area, including the overlapping (grey green) and nonoverlapping (yellow) areas.
(A)

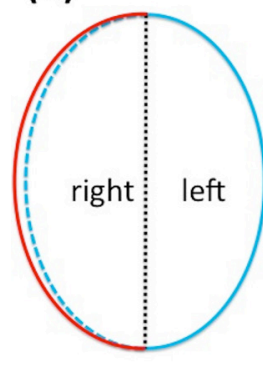

(B)

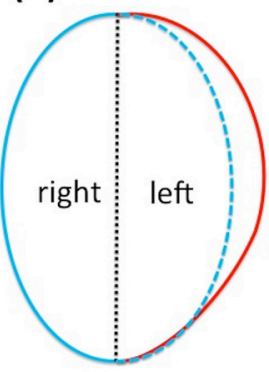

(C)

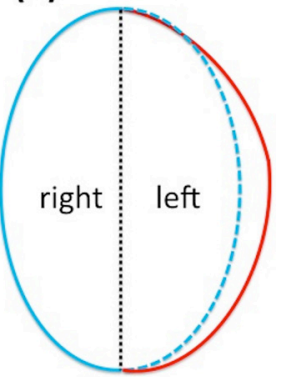

Figure 3 - Schematic illustration of the computed tomography images of asymmetric crania to predict the laterality of chronic subdural hematoma. (A and B) In left-sided hematoma, cranial asymmetry presents with a lower cranial index of symmetry (CIS) ratio and larger left-sided nonoverlapping areas. C) Right-sided hematoma more commonly occurs in symmetric crania with a high CIS ratio and slightly larger nonoverlapping areas. Red line - hematoma side, black dotted line - midline, blue line - opposite side of hematoma, blue dotted line horizontal flip on image to the opposite side.

we determined the angles in the occipital vaults, using a method similar to that of Lee et $\mathrm{al}^{4}$ and Kim et $\mathrm{al},{ }^{6}$ and only unilateral CSDH patients were enrolled here. The present results indicate that flat cranial asymmetry was not significantly associated with $\mathrm{CSDH}$ laterality, indicating that the laterality of $\mathrm{CSDH}$ does not contribute to cranial flatness, as determined through flat angle measurements.

Cranial morphology may not always be similar between the right and left hemispheres. ${ }^{5,6,13}$ Although determination of the angle on the cranium is a simple method, these angles cannot accurately reflect cranial asymmetry. Based on computerized simulations, cranial symmetry may be assessed using an objective semiautomated method. ${ }^{7}$ After defining the midline 
with 2 dots, the intracranial vaults at the right and left sides can be analyzed by encoding the number of pixels. The overlapping and nonoverlapping areas can be compared and calculated through horizontally flipping. Here, most patients (86.7\%) displayed left-sided predominance in the nonoverlapping areas. The CIS ratio was higher in patients with right-dominant nonoverlapping areas than in those with left-dominant nonoverlapping areas. Patients with right-dominant nonoverlapping area more commonly displayed right-sided $\mathrm{CSDH}$, whereas those with left-dominant nonoverlapping areas more frequently presented left-sided CSDH, indicating that most patients had a left-sided predominant cranial asymmetry and a higher risk of left-sided CSDH. Otherwise, left sided CSDH is commonly among higher asymmetric crania with a low CIS ratio, whereas symmetric crania with a high CIS ratio may predict right-sided $\mathrm{CSDH}$. Our results support the hypothesis that CSDH laterality contributes to the severity of asymmetric crania.

This study has some limitations. First, our sample was relatively small. Second, this was a retrospective study, only including patients receiving surgical intervention for CSDH. Third, differences between bilateral and unilateral $\mathrm{CSDH}$ were not compared using the objective semiautomated method developed here. Further prospective studies with larger samples are required to confirm the present findings.

In conclusion, although most patients with unilateral CSDH present with right-sided hematoma, this study compared the conventional angle method and the objective semiautomated method developed here to assess CSDH laterality. This study shows that high cranial asymmetry with a low CIS ratio potentially predicts left-sided CSDHs, whereas right-sided CSDHs are potentially more common in symmetric crania with a high CIS ratio. The present results indicate that the laterality of $\mathrm{CSDH}$ is potentially associated with the severity of cranial asymmetry.

\section{References}

1. Hsieh CT, Su IC, Hsu SK, Huang CT, Lian FJ, Chang CJ. Chronic subdural hematoma: Differences between unilateral and bilateral occurrence. J Clin Neurosci 2016; 34: 252-258.

2. MacFarlane MR, Weerakkody Y, Kathiravel Y. Chronic subdural haematomas are more common on the left than on the right. $J$ Clin Neurosci 2009; 16: 642-644.

3. Nayil K, Ramzan A, Sajad A, Zahoor S, Wani A, Nizami F, et al. Subdural hematomas: an analysis of 1181 Kashmiri patients. World Neurosurg 2012; 77: 103-110.

4. Lee KS, Bae WK, Yoon SM, Doh JW, Bae HG, Yun IG. Location of the chronic subdural haematoma: role of the gravity and cranial morphology. Brain Inj 2001; 15: 47-52.

5. Akhaddar A, Bensghir M, Elmoustarchid B, Abouqal R, Boucetta M. Influence of cranial morphology on the location of chronic subdural haematoma. Acta Neurochir (Wien) 2009; 151: 1235-1240.

6. Kim BG, Lee KS, Shim JJ, Yoon SM, Doh JW, Bae HG. What determines the laterality of the chronic subdural hematoma? $J$ Korean Neurosurg Soc 2010; 47: 424-427.

7. Zonenshayn M, Kronberg E, Souweidane MM. Cranial index of symmetry: an objective semiautomated measure of plagiocephaly. Technical note. J Neurosurg 2004; 100: 537-540.

8. Adhiyaman V, Chattopadhyay I, Irshad F, Curran D, Abraham $S$. Increasing incidence of chronic subdural haematoma in the elderly. QJM 2017; 110: 375-378.

9. Rauhala M, Luoto TM, Huhtala H, Iverson GL, Niskakangas T, Ohman J, et al. The incidence of chronic subdural hematomas from 1990 to 2015 in a defined Finnish population. J Neurosurg 2019: 1-11.

10. Yang W, Huang J. Chronic Subdural Hematoma: Epidemiology and Natural History. Neurosurg Clin NAm 2017; 28: 205-210.

11. Yang AI, Balser DS, Mikheev A, Offen S, Huang JH, Babb J, et al. Cerebral atrophy is associated with development of chronic subdural haematoma. Brain Inj 2012; 26: 1731-1736.

12. Edlmann E, Giorgi-Coll S, Whitfield PC, Carpenter KLH, Hutchinson PJ. Pathophysiology of chronic subdural haematoma: inflammation, angiogenesis and implications for pharmacotherapy. J Neuroinflammation 2017; 14: 108.

13. Pesce Delfino V, Potente F, Chiarelli B. [Evaluation of skull vault asymmetry using methods of analytical morphometry]. Boll Soc Ital Biol Sper 1990; 66: 405-411. [Italian] 